\title{
Herbal medicines: great applause and less clinical use
}

\author{
Volume 2 Issue 4 - 2015
}

\section{Opinion}

Herbal medicine has been believed in, and applied since thousands of years. People are mostly familiar with certain grandmother's preparations of herbal medicines for common ailments, like cold, toothache and headache. Being easy to prepare via infusion or macerating, etc., herbal medicine have been very popular, particularly in rural areas, and among poor people unable to afford costly chemical drugs. However, recently, herbal medicine has gained great attention from scientists worldwide, and numerous published papers in scientific journals, and scientific meetings have their main topics more and more focusing on investigating plants' efficacious ingredients. Not only in China and India, where traditional Chinese medicine and Ayurveda, respectively, have gained great interest, but it has also been a hot research topic in other countries all over the world. Numerous in vitro and in vivo experiments have been conducted on herbal medicines, and they yielded great positive results that suggest that many natural compounds could be potential candidates for the development of new effective drugs for patients suffering from challenging diseases, particularly those named as multifactorial or modern diseases, which are lacking effective treatment.

However, most of those studies, which are in some cases, extended to certain clinical trials, are still not providing developed and commercialized drugs to be available for the patient compared to the chemical medicines. Herein, I would like to draw attention to the importance and the urgent need to take more serious and quicker steps on the research on herbal medicines in the purpose of development into new medicines, rather than being limited to publish original papers, or conduct very limited clinical trials. New drug discovery has become an urgent requirement, particularly with the recent modern life challenges including environment pollution, unhealthy diet and lifestyle, study and work pressure, etc. All these factors could lead to the increase of many diseases prevalence, such as different types of cancer. The drugs available in the market are still unable to face these challenges successfully, hence the need for another source for discovering new effective drugs. Believed to be safer and hopeful, herbal medicine has been regarded as the key element to overcome these challenging health problems.

Many researchers in different fields of pharmaceutical research have been searching among the rich dictionaries of traditional medicine for potential natural drugs. Actually numerous herbal medicines and herbal compounds have been proven to be highly efficient and safe for the treatment of many diseases, and this was proven experimentally in vitro, in vivo and through clinical studies. For instance Davaie Loban, ${ }^{1}$ resveratrol ${ }^{2}$ and many other natural plants have shown many auspicious results towards the treatment of Alzheimer's disease, and Thymoquinone for the prevention and the treatment of cancer. ${ }^{3}$ Many other examples can be found easily through reading scientific papers published in different journals published worldwide, in different medicinal fields about different ailments and diseases.

Indeed, herbal molecules possess many properties that provide them with a prestigious position among potential candidates for new drug discovery. For instance they provide the researcher with primary

\author{
Besma Boubertakh ${ }^{1,2}$ \\ 'University of Chinese Academy of Sciences, Kunming Institute \\ of Zoology, China \\ ${ }^{2}$ Key Laboratory of Animal Models and Human Disease \\ Mechanisms of Chinese Academy of Sciences \& Yunnan Province, \\ Kunming Institute of Zoology, Kunming, Yunnan 650223, PR \\ China
}

Correspondence: Besma Boubertakh, University of Chinese Academy of Sciences, Kunming Institute of Zoology, Key Laboratory of Animal Models and Human Disease Mechanisms of Chinese Academy of Sciences \& Yunnan Province, Kunming, Yunnan Province, 650223, China, Email www.boubertakhbesma@outlook.com

Received: October 16, 2015 | Published: October 17, 2015

information about their potential activity through consulting their use in folk medicine, and they have been proven to be effectively safer than many synthetic compounds. In addition, they present a rich and greatly wide source for the discovery of new drugs since a wide variety of herbal plants grow in different regions and different seasons. These reasons are among many others that enhance the importance of herbal compounds in expediting the pharmaceutical discovery of new treatment. ${ }^{4}$ Although all the promising findings about herbal medicines, they are still not provided for the patients as a treatment. That is why I encourage and recommend that scientific researchers and pharmaceutical companies should focus more on establishing the full pharmacological and toxicological profiles of potential natural compounds, and on conducting more clinical studies on these auspicious candidates, in order to provide the patient, as quick as possible, with an effective, yet safer drug, which could help the patients to promote their health state, and help further leading to positive economical and social impact globally.

\section{Acknowledgements}

None.

\section{Conflicts on interest}

The author declares that there is no conflict of interest.

\section{Funding}

None.

\section{References}

1. Haleh T, Rostam S, Rasool C, et al. Herbal medicine Davaie Loban in mild to moderate alzheimer 's disease: a 12-week randomized doubleblind placebo-controlled clinical trial. Complementary Therapies in Medicine. 2015;23(6):767-772. 
2. Giulio P, Jun W, Lap h, et al. Roles of resveratrol and other grapederived polyphenols in alzheimer's disease prevention and treatment Biochimica et Biophysica Acta (BBA)-Molecular Basis of Disease. 2015;1852(6):1202-1208.

3. Juthika K, Kyung-Soo C, Okezie IA, et al. Mechanistic perspectives on cancer chemoprevention/chemotherapeutic effects of thymoquinone Mutation Research/Fundamental and Molecular Mechanisms of Mutagenesis. 2014;768:22-34.
4. Ghanemi A, Boubertakh B. Shorter and sturdier bridges between traditional Chinese medicines and modern pharmacology. Saudi Pharma J. 2015;23(3):330-332. 